\title{
Age-related changes in brain activation during a delayed item recognition task
}

\author{
Eric Zarahn*, Brian Rakitin, Diane Abela, Joseph Flynn, Yaakov Stern \\ Cognitive Neuroscience Division, Taub Institute, P and S Box 16, 630 West 168th Street, Columbia University, NY 10032, USA
}

Received 5 August 2005; received in revised form 9 February 2006; accepted 3 March 2006

Available online 18 April 2006

\begin{abstract}
To test competing models of age-related changes in brain functioning (capacity limitation, neural efficiency, compensatory reorganization, and dedifferentiation), young $(n=40$; mean age $=25.1$ years $)$ and elderly $(n=18$; mean age $=74.4$ years $)$ subjects performed a delayed item recognition task for visually presented letters with three set sizes $(1,3$, or 6 letters) while being scanned with BOLD fMRI. Spatial patterns of brain activity corresponding to either the slope or $y$-intercept of fMRI signal with respect to set size during memory set encoding, retention delay, or probe stimulus presentation trial phases were compared between elder and young populations. Age effects on fMRI slope during encoding and on fMRI $y$-intercept during retention delay were consistent with neural inefficiency; age effects on fMRI slope during retention delay were consistent with dedifferentiation. None of the other fMRI signal components showed any detectable age effects. These results suggest that, even within the same task, the nature of brain activation changes with aging can vary based on cognitive process engaged.

(C) 2006 Elsevier Inc. All rights reserved.
\end{abstract}

Keywords: Aging; Parietal cortex; Prefrontal cortex; Premotor cortex; Verbal working memory; Working memory; Articulatory loop; Memory load; Compensatory reorganization; Neural efficiency; Capacity limitation; Dedifferentiation; Canonical variates analysis

\section{Introduction}

Four extant hypotheses concerning changes in brain function with aging are compensatory reorganization, dedifferentiation, computational capacity limitation, and neural inefficiency. The purpose of the current paper is to test the ability of these hypotheses to predict age-related changes in brain function associated with various aspects of cognitive processing, including verbal working memory (WM) maintenance, engaged during performance of a delayed item recognition (DIR) task for letters.

\subsection{Brain areas implicated in working memory maintenance in the young}

WM is a psychological construct used to describe the maintenance and manipulation of information on a time scale

\footnotetext{
* Corresponding author. Tel.: +1 212305 7855; fax: +1 2123421838 .

E-mail address: ez84@columbia.edu (E.Zarahn).
}

of seconds [7]. WM seems to be divided into verbal, spatial, and object sub-systems $[8,37,82,94]$. Verbal WM is thought to be critical for language comprehension and reasoning [5]. Based on neuropsychological dissociations $[95,98]$ and word length, phonemic similarity, irrelevant speech, and articulatory suppression effects $[6,11,22,51]$, the maintenance of information in verbal WM has been modeled as an articulatory loop in which sub-vocal rehearsal refreshes a phonological store. Experimental variation of the amount of information to be stored in verbal WM (WM load) has yielded findings of increases in fMRI signal in premotor, parietal, inferior frontal, and middle frontal areas $[55,72,73,76,96]$. At least some aspects of articulatory loop neural processing vary in intensity with WM load $[45,96,106]$.

\subsection{Age-related changes in the brain mechanisms of verbal WM}

Even in the absence of Alzheimer's disease (AD) and other recognized brain diseases, aging is associated with 
impairment in several different memory variables [78], including WM $[9,17,23,49]$. In particular, load-dependent deficits in WM processing have been observed with normal aging $[2,23,26,62,64]$. Age-related deficits in cognition are assumed to stem from age-related brain pathology [88]. Normal aging is associated with a decrease in neuropil and neuronal number in cortex $[12,21,25]$ and in the subiculum region of the hippocampus $[84,99]$, an increase in the number of infarcts in cortex, basal ganglia, and white matter [65], an increase in MRI white matter lesions [80], an increase in density of neurofibrillary tangles in the CA1 region of the hippocampus [81], and a global decrease in gray matter volume [31].

There is the broad question of whether the functional neural circuitry of the brain remains static in the face of this neuropathology. Though not exhaustive, four extant hypotheses concerning changes in brain function with normal aging are compensatory reorganization, dedifferentiation, computational capacity limitation, and neural inefficiency. The purpose of the current study is to test the ability of these hypotheses to predict age-related changes in brain function associated with load-dependent and load-independent aspects of encoding, storage/rehearsal, and recognition/response components of a DIR task for letters [90], which is thought to tap verbal WM maintenance. These four hypotheses will now be briefly described, in turn.

\subsection{Models which predict changes in patterns of brain activation with aging}

Some have put forward a hypothesis that the brain is constructed such that it can in some sense compensate for neuropathology (such as that associated with normal aging) via macro-reorganization of neural circuits $[4,10,14,33,87,100]$. The teleological argument is that the effect of this reorganization would be to reduce or potentially even eliminate any behavioral consequences of the neuropathology that would otherwise occur. Compensatory reorganization, occurring to varying degrees across individuals, could potentially explain how age-associated neuropathology exists even in certain proportions of the non-demented elderly $[34,81,83,85]$, and why variability in cognitive functioning increases with age [18]. Consistent with a special version of the compensatory reorganization hypothesis referred to as HAROLD (hemispheric asymmetry reduction in older adults [15]), a more bilateral PFC fMRI activation pattern in older adults than younger adults has been reported in word encoding [60,71], source memory [14], retrieval [53], working memory $[15,69]$, and visual attention task contexts [15].

The types of compensatory reorganization models that we consider here (subsuming, but not limited to, the HAROLD model) posit that higher performing elders are higher performing because of a change in brain reorganization relative to both young subjects and lower performing elders. Therefore, under this type of compensatory reorganization there would be a cross-sectional correlation within elders between the degree of brain reorganization and performance, such that the brain activation patterns of higher performing elders would be more dissimilar than those of lower performing elders to young subject activation patterns [14]. We refer to all such models as cross-sectional compensatory reorganization models, to distinguish them from other types of compensatory models which do not require such cross-sectional correlations [89]. The current work can only weakly test the latter type of models, so we focus on testing cross-sectional compensatory reorganization models.

Dedifferentiation is another hypothesis that predicts nonidentical brain activity patterns between young and elder populations. But, unlike compensatory reorganization, this change is not beneficial for the behavior in question, and is thought to represent a general deterioration in the integrity of brain circuitry [15]. Dedifferentiation and compensatory reorganization can be distinguished as the two make opposite predictions concerning the cross-sectional relationship of age-related differences in activation patterns and performance.

A critical notion concerning both of these theories is that the spatial pattern of neuronal activity in a brain that has been reorganized or de-differentiated is not identical to within a scaling factor to the corresponding canonical pattern of brain activation (in our case, that of the healthy, young population). From here on, the phrase "identical patterns" implicitly means identical to within a scaling factor. In Section 1.5, we discuss the method used to test whether elder and young activation patterns are identical.

\subsection{Models which predict no change in patterns of brain activation with aging}

Another general hypothesis regarding the effect of neuropathology on brain function is a reduction in the capacity of information representation or throughput in a brain circuit. This might perhaps be caused by a limitation on the amount or quality of information entering a brain circuit due to impairment in sensory systems $[35,47,50]$. A simple reduction in computational capacity would predict, in the context of identical task stimuli and instructions, a decrease in both performance and neurophysiologic activity (i.e., less total ionic flux across neuronal membranes due to synaptic transmission, therefore less ATP utilization through ionic pumps, and presumably less cerebral blood flow), and so would arguably be associated with identical brain activity patterns in young and elders. Reductions in activation with aging have been reported in anterior frontal cortex [32,57,79], dorsolateral PFC [41,43,58,74,75], hippocampus [57,59], anterior cingulate [58], temporal [40], parietal [40,58] and occipital cortices $[15,33,40,52,57]$. Some of these reduced activations have been associated with age-related impairments in certain cognitive functions, such as resolution of competing response impetuses [43], memory scanning speed [74], and feature binding [59]. 
Often, age-related decreases in activation in certain areas have been found concomitantly with age-related increases in other areas $[15,33,57,58,75]$, which is inconsistent with a simple capacity limitation hypothesis. Increases in brain activation in a behaviorally impaired group have been sometimes conceived as a reduction in neural efficiency [16,76]. Here, we define neural efficiency as the amount of performancerelevant computational work (operationalized here as measures of behavioral performance) performed per unit of synaptic activity (operationalized here as BOLD fMRI signal change). We also define neural inefficiency (which might be a more stable measure than neural efficiency) as simply the reciprocal of neural efficiency. We consider neural (in)efficiency as being not the property of individual regions, but as a property of a brain system/circuit. For example, if elders show lowered performance on average compared to young subjects and engage the same brain system during task performance but to a greater degree, then we would say that the elders' brain system is less efficient than that of young subjects. Neural efficiency, like capacity limitation, would be associated with identical patterns of brain activation in young and elders.

\subsection{Comparing patterns of brain activation}

We have established a dichotomy between models of agerelated brain activation change with respect to changes in brain activation patterns. On the one hand, compensatory reorganization and de-differentiation both predict (on average) different activation patterns in young and elders. On the other, neural inefficiency and capacity limitation both predict identical activation patterns in young and elders. Obviously, then, discrimination between these two pairs of hypotheses requires some sort of test as to whether young and elder brain activation patterns are identical.

Certain approaches that have been used previously to compare patterns of brain activation have caveats. Direct comparison of voxel-wise signal intensities between groups via statistical parametric mapping (SPM; [15]) is an ambiguous test of identical spatial patterns, as even a pure scaling could lead to the existence of true voxel-wise intensity differences (Fig. 1). Also, visual comparison of group [14,32] or condition-specific [73] thresholded statistical maps suffers from the same problem [77] (Fig. 1). An additional difficulty of this latter method is that substantial variability between realizations of thresholded maps would be expected due to intermediate levels of statistical power [20,70]. Finally, region of interest laterality indices [13], though providing a valid test of identical patterns of region of interest effects in noiseless data, can be exceedingly unstable in practice due to their involving ratios of estimated activation; moreover, they do not compare entire brain activation patterns.

To test for non-identity of young and elder fMRI activation patterns, we used sequential latent root testing in the context of a canonical variates analysis (CVA) for imaging data with spatially correlated errors [103]. Unlike partial least-squares

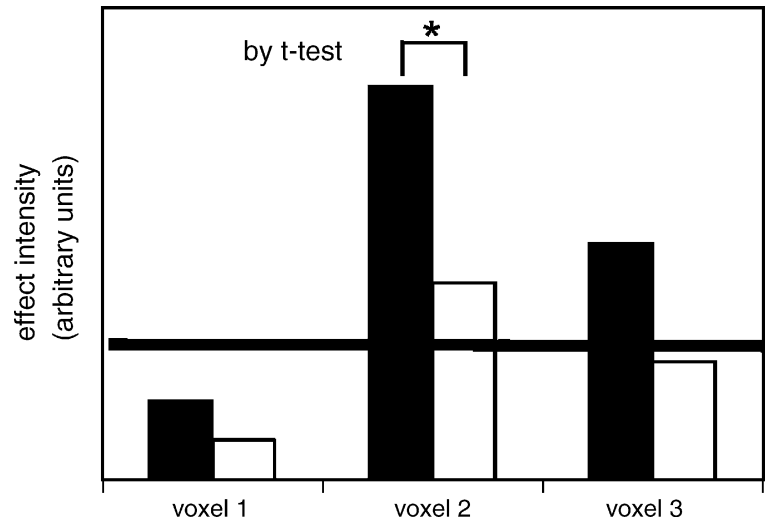

Fig. 1. In this hypothetical, groups A ( $\mathbf{\square})$ and B ( $\square$ ) express identical brain activation patterns, with the expression of this pattern in group A being twice that in group B. If visually comparing thresholded maps between groups (threshold indicated by the black horizontal line), one might incorrectly conclude differing patterns of activation in the two groups because of the restriction of suprathreshold activation in voxel 3 to group A. A related inferential problem can arise when directly comparing voxel-wise intensities between two groups via $t$-tests/ANOVA, in which case one might incorrectly conclude differing patterns of activation in the two groups because only voxel 2 ("indicating high probability to detect a group difference via $t$-test) tends to be detected as having a difference in intensity between groups A and B. Both of these problems spring from the fact that neither visual inspection of thresholded maps nor group comparisons via $t$-test formally assess the hypothesis of spatial patterns of brain activation being identical to within a scaling factor.

[56], this CVA method is invariant to linear transformations of the predictor variables and provides parametric distributional approximations that are valid for correlated observations, as is the case in our repeated measures design [103].

\section{Methods}

\subsection{Study population}

Forty healthy, young subjects (31 M and $9 \mathrm{~F}$; mean (S.D.) age $=25.1$ (3.9); mean years of education $=15.7$ (1.4); all right handed), recruited through flyers posted at the Columbia University campus and advertisements placed in local newspapers, and 18 healthy, elderly subjects recruited from senior centers in the New York City area (7 M and 11 F; mean (S.D.) age $=74.4$ (6.9); mean (S.D.) years of education $=15.3(2.4)$; all right handed) participated. Global cognitive functioning was assessed with the mMMSTotal [86], and all subjects were classified as non-demented and without serious cognitive impairment (elder mean mMMSTotal = 53.3 (2.5); young mean mMMSTotal=55.1 (1.5)). IQ was estimated with the North American Reading Test (NART), to test if there was a systematic difference between groups in IQ [88]; the elder NART scores were significantly lower than the young (elder mean NARTIQ = 116.8 (6.4); young mean NARTIQ = 120.3 $(6.1) ; t(56)=2.02$, two-tailed $p=0.049)$. All subjects supplied informed consent. Volunteers were screened for psychiatric and neurologic illness via a questionnaire. The current 


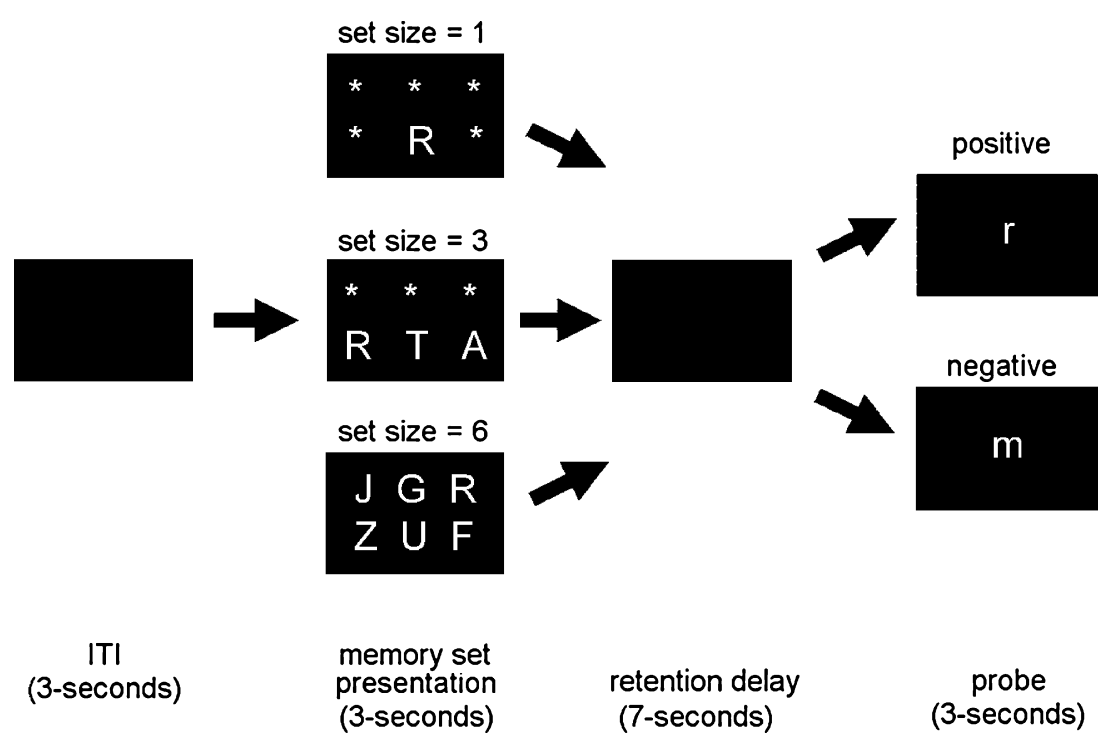

Fig. 2. The delayed item recognition task is schematized.

young subject dataset has been used in a previous report to examine load-dependent patterns of retention delay fMRI signal [106].

Age and gender were confounded in this experiment $(t(56)=3.04$, two-tailed $p=0.004)$. To eliminate main effects of gender from the estimation of age effects, gender was included as a covariate in the imaging analysis.

\subsection{Behavioral task}

The behavioral task used during fMRI scanning was a DIR task for letters [90]. The task is schematized in Fig. 2. Task and training details are provided in [106]. The critical factor was set size, which is the number of letters (either 1,3 , or 6) to be remembered on each trial. Set size was varied pseudo-randomly across trials. Each of three experimental blocks contained 10 trials at each of the three set sizes, with five true negative and five true positive probes per set size, yielding a total of 30 trials per set size per subject.

\section{3. fMRI data acquisition}

During the performance of each block of the DIR task, 207 BOLD images $[48,63]$, were acquired with an Intera 1.5 T Phillips MR scanner equipped with a standard quadrature head coil, using a gradient echo echo-planar (GE-EPI) pulse sequence $\left(\mathrm{TE} / \mathrm{TR}=50 \mathrm{~ms} / 3000 \mathrm{~ms}\right.$; flip angle $=90^{\circ}$; $64 \times 64$ matrix, in-plane voxel size $=3.124 \mathrm{~mm} \times 3.124 \mathrm{~mm}$; slice thickness $=8 \mathrm{~mm}$ (no gap); 17 trans-axial slices per volume). Four additional GE-EPI excitations were performed before the task began, at the beginning of each run, to allow transverse magnetization immediately after radio-frequency excitation to approach its steady-state value; the images corresponding to these excitations were discarded. A T2-weighted, fast spin echo image was also acquired from each subject for spatial normalization purposes $\left(\mathrm{TE} / \mathrm{TR}=100 \mathrm{~ms} / 2000 \mathrm{~ms}\right.$; flip angle $=90^{\circ}, 256 \times 256$ matrix; in-plane voxel size $=0.781 \mathrm{~mm} \times 0.781 \mathrm{~mm}$; slice thickness $=8 \mathrm{~mm}$ (no gap); 17 trans-axial slices per volume).

Task stimuli were back-projected onto a screen located at the foot of the MRI bed using an LCD projector. Subjects viewed the screen via a mirror system located in the head coil. Responses were made on a LUMItouch response system (Photon Control Company). Task onset was electronically synchronized with the MRI acquisition computer. Task administration and collection of RT and accuracy data were controlled using PsyScope [19].

\section{4. fMRI data pre-processing}

All image pre-processing was implemented using the SPM99 program (Wellcome Department of Cognitive Neurology). The following steps were taken in turn for each subject's GE-EPI dataset: data were temporally interpolated and shifted to correct for the order of slice acquisition, using the first slice acquired in the TR as the reference. All GE-EPI images were realigned to the first volume of the first session. The T2-weighted (structural) image was co-registered to the first EPI volume using the mutual information co-registration algorithm implemented in SPM99. This co-registered highresolution image was then used to determine parameters $(7 \times 8 \times 7$ non-linear basis functions) for transformation into a Talairach standard space [92] defined by the Montreal Neurologic Institute (MNI) template brain supplied with SPM99. This transformation was then applied to the GEEPI data, which were re-sliced using sinc-interpolation to $2 \mathrm{~mm} \times 2 \mathrm{~mm} \times 2 \mathrm{~mm}$. 


\section{5. fMRI time-series modeling}

All statistical analysis was implemented using the SPM99 program and other code written in MATLAB 5.3 (Mathworks, Natick MA). The fMRI data analysis comprised two levels of voxel-wise GLMs [29]. In the (subject-separable) first-level GLM, the GE-EPI time-series were modeled with regressors representing the expected BOLD fMRI response (implicitly, relative to the blank intervals) to the three DIR trial components of memory set presentation, retention delay, and probe presentation/response, separately for each crossing of the set size and true positive/true negative factors (trials on which there was no motor response from the subject during the probe period were modeled separately, and were not included for analysis at the second-level GLM). The regressors were constructed by convolutions of an indicator sequence (i.e., a train of discrete-time delta functions) representing DIR trial component onsets, an assumed BOLD impulse response function (as represented by default in SPM99), and a rectangular function of duration dictated by the duration of the assumed neural response [105]. Based in part on prior knowledge as well as regression diagnostics, two rectangular functions (and hence, two regressors) were used for the trial components of memory set presentation and probe presentation: one modeling a relatively brief $(400 \mathrm{~ms})$ neural response at the beginning of that trial component, and another modeling a neural response lasting throughout that entire component $(3000 \mathrm{~ms})$; a single rectangular function of $7000 \mathrm{~ms}$ duration was used for the retention delay.

For both the memory set presentation and probe presentation trial components, a linear combination (i.e., a contrast) of the two parameter estimates was computed which estimated the area under the curve of the neural response for that trial component. For the retention delay trial component, the analogous contrast was simply the coefficient of its single basis function. These contrast estimate images were intensity normalized via voxel-wise division by the time-series mean, masked with an image that represented the intersection of useable data from all subjects and had a gray matter prior probability $>0.25$ in standard space using the prior probability images supplied with the SPM99 program (this eliminated the ventricles and surrounding white matter from the search volume), and spatially smoothed with an isotropic Gaussian kernel (full-width-at-half-maximum $=8 \mathrm{~mm}$ ). The purpose of masking prior to smoothing is to avoid differences between subjects in partial volume contributions to data within the mask after smoothing. The resulting images were used as the dependent data in a second-level, voxel-wise GLM [38] that modeled 18 repeated measures per subject per voxel, with a design matrix representing two between-subjects factors (age and gender) and three repeated measure factors (trial component, set size, and probe type). Certain sets of effects of interest (see Section 2.6) from this second-level GLM were subjected to sequential latent root testing. The repeated measures covariance matrix of the second-level data was estimated at each voxel and spatially averaged. This matrix was used to approximate the known observation error covariance matrix ( $\Sigma$ in [103]). Results concerning gender are not reported here, but its inclusion as a factor meant that estimates of age effects controlled for gender.

\subsection{Sequential latent root testing}

In this version of CVA [103], a singular value decomposition (SVD) is performed on the whitened effects of interest, followed by sequential latent root testing (with $\alpha$ controlled at a desired level) to assess the number of latent spatial patterns. The resulting statistical tests use parametric approximations ( $F$-statistics), which have been validated using computer simulations [103]. The term "effects of interest" refers to a $k$-dimensional subspace of the design matrix of relevance for a particular hypothesis. Based on our interest in neural responses that increased with WM load as well as those that were independent of WM load, effects of interest comprised all six combinations of slopes and $y$-intercepts of fMRI signal with respect to set size with the three trial components and with all separately representing elder and young (thus $k=2$ for all of six sets of effects of interest). Effects of interest were averaged over the probe type factor (i.e., true positive and true negative trial types), thus making the effective number of trials per subject per set size equal to 30 (or close to 30 when accounting for no-response trials).

Significant latent spatial patterns are presented for descriptive purposes scaled by their singular values (analogous to SPM $\{t\}$ images [103]), thresholded for descriptive purposes at a $t$ value corresponding to $p<0.001$ uncorrected for multiple comparisons and a cluster size of 50 voxels. This threshold does not control map-wise statistical significance at $\alpha=0.05$ $[28,101]$, and so is only meant to provide a somewhat condensed description of the significant latent spatial patterns.

The signs of the voxel values in a latent spatial pattern and its corresponding expression across subjects (or groups) are only meaningful in their product (i.e., the signs of each in isolation may be thought of as completely arbitrary). One multiplies a particular latent spatial pattern by its predicted expression to yield the predicted contribution from that latent pattern to the effects of interest [103].

\subsection{SPM voxel-wise intensity tests}

Using the same second-level GLM as in the CVA analysis, SPM was used to test for voxel-wise intensity differences between young and elder groups in the fMRI slopes and fMRI $y$-intercepts in all three trial components. The statistical threshold was selected to control $\alpha$ at 0.1 per two-tailed effect, corrected for multiple voxel-wise comparisons using Gaussian random field theory [101].

\subsection{Neural inefficiency}

The neural inefficiency of a given latent pattern expression in each subject was computed by dividing the observed 


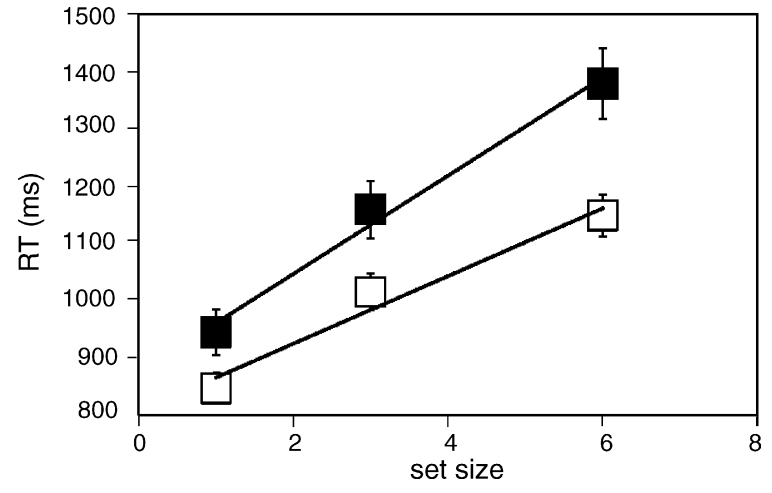

Fig. 3. The relationship of reaction time (RT) to set size is plotted separately for elder $(\square)$ and young $(\square)$ subjects. The lines are least-squares fits. Error bars are estimated standard errors, and so reflect sample sizes $\left(n_{\text {young }}=40\right.$, $\left.n_{\text {old }}=18\right)$ as well as standard deviations.

expression in each subject by a performance value (where a higher performance value means better performance). The performance values used to compute neural inefficiency were a measure of accuracy independent of response bias $\left(d^{\prime}\right)$ at set size 6 (age effects on discriminability were expected to be largest at the largest set size), RT slope ${ }^{-1}$, and RT intercept ${ }^{-1}$.

\section{Results}

\subsection{Reaction time}

As expected [90], reaction time was affected by set size in both young $(F(1.8,68.9)=106.2, p<0.001)$ and elder $(F(1.7,28.0)=77.8, p<0.001)$ subjects (Fig. 3$)$. The interaction of age group and set size was significant $(F(1.7$, $96.1)=6.1, p=0.005)$. Relatedly, a direct comparison of RT slopes revealed a significantly larger slope in elders (59.0 (30.8) $\mathrm{ms} / \mathrm{letter}$ in young, 85.7 (35.7) $\mathrm{ms} / \mathrm{letter}$ in elders; $t(56)=-2.91$, two-tailed $p=0.005)$. The variability of RT slope in the elder group was not significantly greater than that in the young group $(F(17,39)=1.35$, $p=0.22$ ).

The $y$-intercepts were not significantly different between young and elders $(806.6(180.0) \mathrm{ms}$ in young, 875.0 (176.1) $\mathrm{ms}$ in elders; $t(56)=1.35$, two-tailed $p=0.183$ ). The

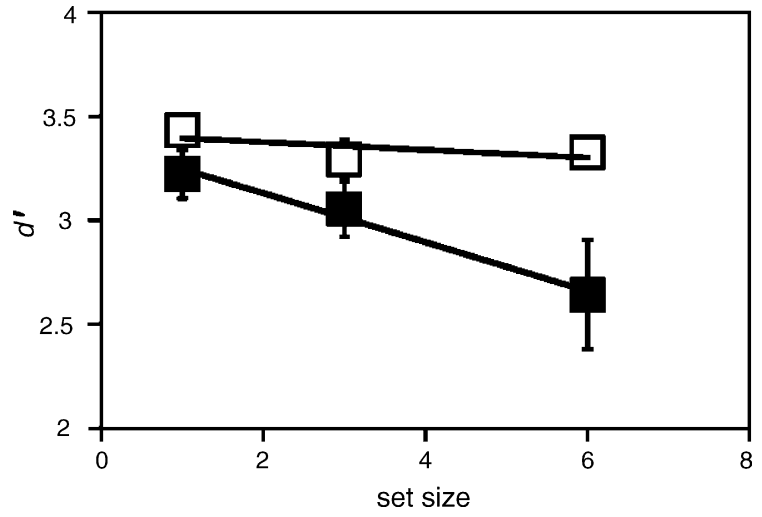

Fig. 4. The relationship of $d^{\prime}$ to set size is plotted separately for elder $(\mathbf{\square})$ and young $(\square)$ subjects. The lines are least-squares fits. Error bars are estimated standard errors, and so reflect sample sizes ( $n_{\text {young }}=40, n_{\text {old }}=18$ ) as well as standard deviations.

variability of RT intercept in the elder group was not significantly greater than that in the young group $(F(17,39)=0.96$, $p=0.52$ ).

\subsection{Accuracy}

While the accuracy of young subjects was not detectably affected by set size $(F(2.0,78)=1.2, p=0.32)$, the accuracy of elder subjects decreased with set size $(F(1.6,27.0)=3.9$, $p=0.04)$, with the interaction of group and set size significant $(F(1.9,103.8)=3.5, p=0.04)$. This suggests that normal aging affects WM capacity (Fig. 4), even though accuracy was quite high in an absolute sense at set size 6 for elder subjects (95\% confidence interval for $d^{\prime}=[2.09,3.20]$ ).

\subsection{DIR task brain activity patterns in young and elders}

At least one latent spatial pattern (the possible outcomes were 0,1 , or 2 patterns) was detected in each of the six sets of effects of interest (Table 1). Two latent spatial patterns were detected only for retention delay activation slope. Thus, the results indicate non-identical WM load-dependent, retention delay activation patterns in young and elder populations and fail to reject identity of activation patterns across age groups in the remaining five sets of effects of interest.

Table 1

Sequential latent root testing results for contrasts representing young and elder brain activity associated with verbal WM task

\begin{tabular}{|c|c|c|c|c|c|}
\hline \multirow[t]{2}{*}{ 2-Dimensional effects of interest } & \multicolumn{2}{|c|}{$\begin{array}{l}\text { Test for at least one } \\
\text { component }\end{array}$} & \multicolumn{2}{|c|}{ Test for two components } & \multirow{2}{*}{$\begin{array}{l}\text { Inferred number } \\
\text { of spatial patterns } \\
\text { at } \alpha=0.05\end{array}$} \\
\hline & $F(540,57137)$ & $p$ & $F(270,38233)$ & $p$ & \\
\hline Load-dependent processing during memory set presentation & 3.51 & $<0.0001$ & 1.07 & 0.22 & 1 \\
\hline Load-dependent processing during retention delay & 1.79 & $<0.0001$ & 1.41 & $<0.0001$ & 2 \\
\hline Load-dependent processing during probe presentation & 1.12 & 0.03 & 0.88 & 0.92 & 1 \\
\hline Load-independent processing during memory set presentation & 2.83 & $<0.0001$ & 0.82 & 0.99 & 1 \\
\hline Load-independent processing during retention delay & 1.22 & 0.0003 & 0.88 & 0.92 & 1 \\
\hline Load-independent processing during probe presentation & 8.96 & $<0.0001$ & 1.03 & 0.37 & 1 \\
\hline
\end{tabular}



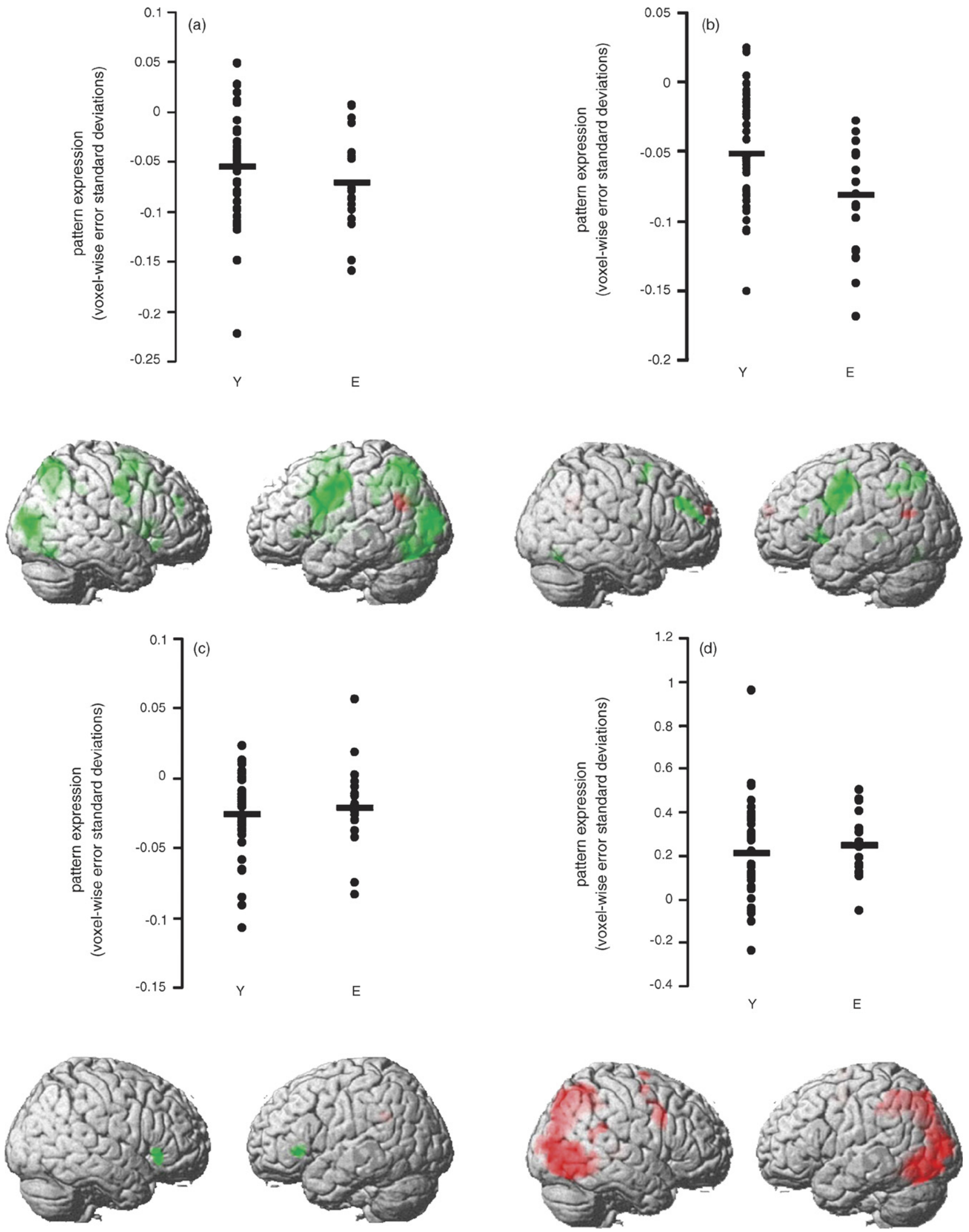

Fig. 5. Scaled first latent spatial patterns (red: positive voxel weights, green: negative voxel weights) and observed (@) and predicted ( $\longrightarrow$ ) subject-wise expressions of the corresponding pattern. (a) slope of fMRI response amplitude vs. set size associated with memory set presentation, (b) slope associated with retention delay, (c) slope associated with probe presentation, (d) $y$-intercept of the relationship of fMRI response amplitude vs. set size associated with memory set presentation, (e) $y$-intercept associated with retention delay, and (f) $y$-intercept associated with probe presentation. See Section 2.6 for thresholding. 


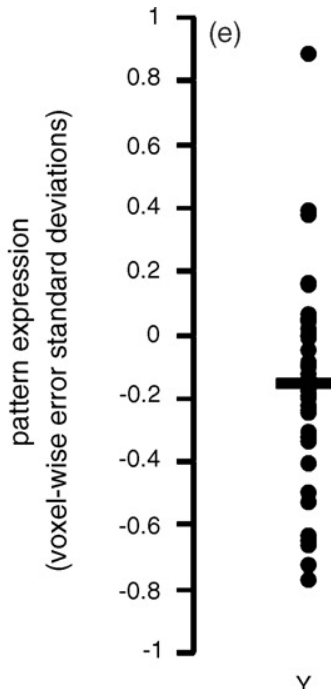

Y

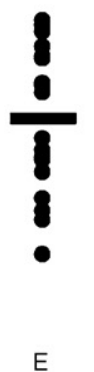

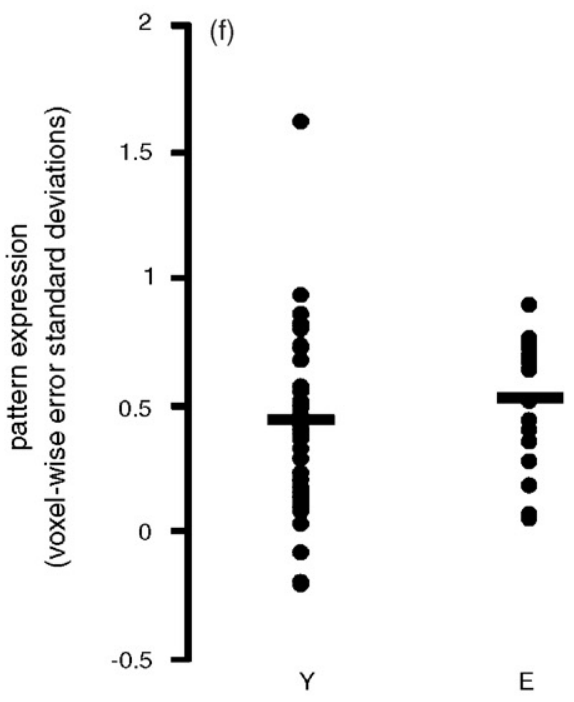
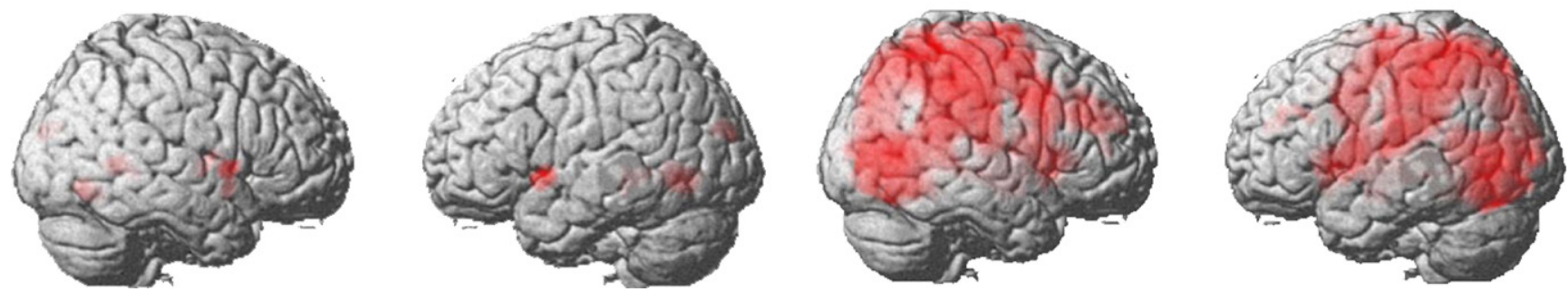

Fig. 5. (Continued).

The first latent spatial patterns and corresponding predicted and observed expressions for all six sets of effects of interest are shown in Fig. 5. We forego a tabular description of the latent spatial patterns in this manuscript because we have used a threshold for descriptive purposes that is more lenient than what should be used to control $\alpha$ at a map-wise level [101], and more fundamentally because such a tabulation would be of limited direct relevance to the hypotheses we wished to test.

\subsection{Compensatory reorganization versus dedifferentiation}

As mentioned above, activation slope patterns corresponding to the retention delay were different in young and elders. The corresponding first latent spatial pattern had the same sign of expression in both age groups (Fig. 5b), and so tends to reflect where both young and elder activate in the same direction, but with a greater magnitude in elders (global maximum of negative spatial weights in left premotor cortex, MNI coordinate $=[-54,-642])$. In contrast, the second latent spatial pattern had opposite signs of expression (elders: negative; young: positive; data not shown) in the two age groups, with elders having a much larger magnitude of expression than young. The only location in the second latent pattern to exceed our display threshold was an area with negative spatial weight in right parahippocampal gyrus/lingual gyrus ([20, -50, 0]; Fig. 6, inset) in which elders had positive (negative spatial weight multiplied by a negative expression) load-dependent retention delay effects and young subjects had essentially nil effects (data not shown). A homologous

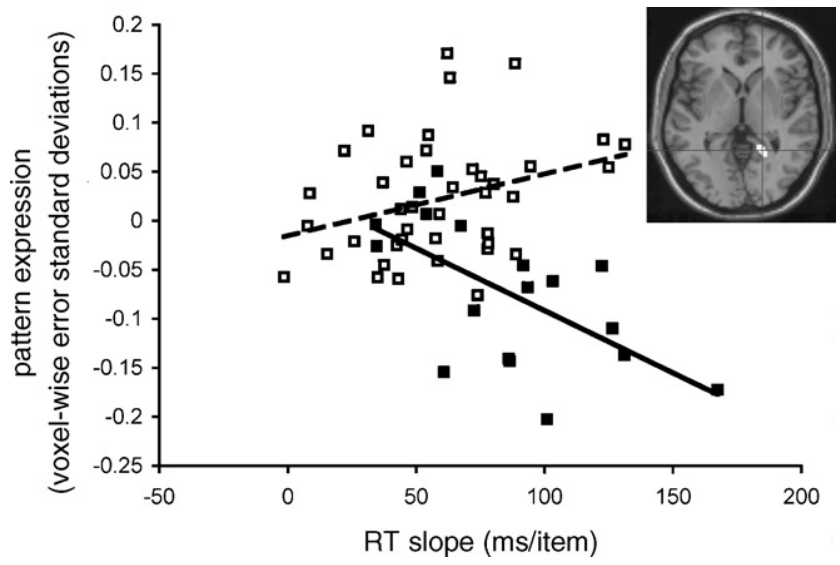

Fig. 6. Observed expressions of latent spatial pattern 2 of load-dependent processing (i.e., fMRI slope) during the retention delay are plotted vs. RT slope; elder $(\mathbf{\square})$, young $(\square)$. An axial slice $(z=0$; neurologic orientation) of the suprathreshold negative spatial weights of latent spatial pattern 2 is shown as an inset. The global maximum is located at right parahippocampal gyrus/lingual gyrus $([20,-50,0])$ at which elder subjects had a positive fMRI slope and young had close to nil fMRI slope. 
Table 2

Comparison between young and elders of neural inefficiency of first latent spatial pattern expressions

\begin{tabular}{|c|c|c|c|}
\hline 2-Dimensional effects of interest & $\begin{array}{l}\text { Age difference in neural } \\
\text { inefficiency with respect } \\
\text { to } d^{\prime} \text { at set size } 6|t(56)|\end{array}$ & $\begin{array}{l}\text { Age difference in neural } \\
\text { inefficiency with respect } \\
\text { to RT slope }|t(56)|\end{array}$ & $\begin{array}{l}\text { Age difference in neural } \\
\text { inefficiency of expression with } \\
\text { respect to RT intercept }|t(56)|\end{array}$ \\
\hline Load-dependent processing during memory set presentation & $2.17^{*}$ & $2.44^{*}$ & 1.53 \\
\hline Load-dependent processing during retention delay ${ }^{\mathrm{a}}$ & 0.55 & $3.89^{*}$ & $2.95^{*}$ \\
\hline Load-dependent processing during probe presentation & 1.04 & 0.17 & 0.24 \\
\hline Load-independent processing during memory set presentation & 0.77 & 0.89 & 1.22 \\
\hline Load-independent processing during retention delay & 0.10 & $4.12^{*}$ & $2.44^{*}$ \\
\hline Load-independent processing during probe presentation & 0.32 & 1.78 & 1.83 \\
\hline
\end{tabular}

* Significant at $\alpha=0.05$, two-tailed, uncorrected for multiple comparisons.

a The brain activation patterns of young and elders for this effect of interest were non-identical, and hence cannot be explained with a pure neural inefficiency model.

area in the left hemisphere with similar effects was evident at the same height threshold but a more lenient cluster threshold.

Parahippocampal cortex is thought to be important for long-term memory [1,107], particularly for recollection [67]. To test if set-size dependent engagement of parahippocampal cortex (and associated areas of the second latent spatial pattern) during the retention delay confers a performance advantage (as would be predicted by cross-sectional compensatory reorganization models), we computed correlations within elders between performance ( $d^{\prime}$ at set size 6 , RT slope, and RT intercept) and expression of this second latent pattern. The only significant correlation was with RT slope ( $R=-0.61$, two-tailed $p=0.007)$. Latent pattern 2 expression for both young and elders converged to zero at the lower (i.e., better performance) end of the range of RT slopes, with expression in elders increasing in magnitude (i.e., getting farther from zero) as RT slope increased (Fig. 6). An important concept here is that when latent pattern 2 expression is zero in both groups, then the groups have identical patterns. This result, greater activation pattern similarity of young to higher performing than to lower performing elder brain activation patterns, is not consistent with cross-sectional compensatory reorganization.

It is also possible that the right parahippocampal gyrus itself is important for compensatory reorganization in some special way that would not be evident in the relationship of latent pattern 2 expression to behavior. All $R^{2}$ estimates between retention delay fMRI slope at the right parahippocampal locus and the behavioral measures in elders were $<0.01$, which is inconsistent with a cross-sectional compensatory role for this brain area in DIR task performance.

\subsection{Neural inefficiency versus capacity limitation}

For none of the effects of interest which had identical activation patterns in young and elders was pattern expression significantly lower in magnitude (i.e., closer to zero) in elder than young subjects (all $p>0.25$ ). This is inconsistent with the capacity limitation hypothesis. For these same effects of interest, neural inefficiency was greater in elders than young subjects for activation slope during encoding with respect to $d^{\prime}$ at set size 6 and RT slope and for activation $y$-intercept during the retention delay with respect to RT slope and RT intercept (Table 2). Also, though not sufficiently explained by the neural inefficiency model (as the young and elder activation patterns were non-identical), the first latent spatial pattern of the activation slope during the retention delay was expressed more inefficiently by elders (RT slope and RT intercept).

\subsection{Age group comparisons via SPM}

Even though SPM could not test the primary hypothesis of interest (namely, whether spatial patterns of activation are identical in elder and young), a reviewer advised that for completeness the results of SPM analyses be presented. Voxel-wise differences between young and elder groups in the slopes or $y$-intercepts of fMRI signal with respect to set size for memory set presentation/encoding, retention delay, and probe presentation were queried with SPM using mapwise corrected thresholds of two-tailed $\alpha=0.1$, which corresponded to a $\left|t_{\text {crit }}\right|=4.49$ with greater than 200 effective degrees of freedom [101]. The null hypothesis of identical activation levels in the two age groups was rejected only for slope during memory set presentation (elder $>$ young), with local maxima in bilateral precentral gyrus (MNI coordinates of $[48,-10,34]$ and $[-40,-14,38])$. We note that young and elder activation patterns were deemed identical by CVA for this set of effects of interest. This provides an empirical proof of the abstract concept presented in Fig. 1.

\section{Discussion}

\subsection{Age effects on brain activation associated with performance of a DIR task}

In the current study, aging was associated with set size dependent decrements in both DIR accuracy and memory scanning speed, which is consistent with previous findings $[2,23,26,62]$. However, as indexed with the $y$-intercept of the RT versus set size relationship, we did not detect an expected 
Table 3

Summary of hypothesis test results

\begin{tabular}{|c|c|c|c|c|}
\hline 2-Dimensional effects of interest & Neural inefficiency & $\begin{array}{l}\text { Capacity } \\
\text { limitation }\end{array}$ & $\begin{array}{l}\text { Compensatory } \\
\text { reorganization }\end{array}$ & Dedifferentiation \\
\hline Load-dependent processing during memory set presentation & Consistent & - & - & - \\
\hline Load-dependent processing during retention delay & - & - & - & Consistent \\
\hline Load-dependent processing during probe presentation & - & - & - & - \\
\hline Load-independent processing during memory set presentation & - & - & - & - \\
\hline Load-independent processing during retention delay & Consistent & - & - & - \\
\hline Load-independent processing during probe presentation & - & - & - & - \\
\hline
\end{tabular}

- means inconsistent with the hypothesis.

overall slowing with aging. Assuming an age effect size on reaction time corresponding to $R=0.50$ [97], power for the current design was $>0.9$ with $\alpha$ controlled at 0.05 .

Nevertheless, in the context of the observed behavioral impairments, we attempted to distinguish between four broad hypotheses concerning what happens to brain function with aging from young (approximately the third decade) to elder (approximately the eighth decade) adulthood. Processes with some linear dependence on WM load were assessed with the slope of fMRI activation amplitude with respect to set size; processes that were engaged even at a theoretical zero WM load (i.e., load-independent processes) were assessed with the $y$-intercept of fMRI activation amplitude with respect to set size; there was no constraint or assumption that such processes are not co-localized in the brain. The findings are summarized in Table 3.

Critical to disambiguating the hypotheses was the concept of identity of brain activation patterns in young and elder populations to within a scaling factor. SPM does not test this; CVA does [103]. However, there is no reason why both CVA and SPM cannot be applied to the same dataset to address different hypotheses. In the current study, very few voxel-wise intensity differences between groups were detected with SPM. This might be surprising given that age effects are commonly reported in the neuroimaging literature. However, most relevant SPM analyses do not use statistical thresholds that control $\alpha$ at a map-wise level, which makes such analyses have higher type I and lower type II error rates than the current one.

Of the six sets of effects of interest examined, five had patterns that were identical (or more precisely, could not be distinguished from identical while controlling $\alpha$ at 0.05) in young and elders. As the power of spatially omnibus tests like those employed with CVA to detect diffuse differences in spatial patterns is better than that of voxel-wise tests [102,103], this finding supports a general similarity of brain activation patterns in young and elders. A visual impression of overall similarity of elder and young activation patterns was noted previously in a study that used a verbal WM task [15].

\subsection{Capacity limitation}

Of the five spatial patterns of activation identical across age groups, none were expressed to a significantly lesser degree in elders than young, and so the idea of an age-related limitation in neurophysiological processing capacity was not supported in the context of DIR for letters. To clarify, this finding does not mean that memory capacity or processing speed do not tend to decrease with age; indeed, they do $[2,23,26,62,97]$. Rather, it means that the data do not support that the upper bound on brain system activation in elders is substantially lower than the corresponding bound in the young [32].

\subsection{Neural inefficiency}

The term neural (in)efficiency has been used to explain the findings of negative relationships between certain electrophysiological and intelligence measures [61,91]. It has also been used in the context of hemodynamic neuroimaging of schizophrenia $[16,54,66]$ and of individual differences in normal populations [76]. In the current study, of the five spatial patterns of activation identical across age groups, two were expressed less efficiently in elders. These were load-dependent processing associated with memory set presentation and load-independent processing associated with the retention delay.

The lower neural efficiency associated with loaddependent memory set presentation in elders seems at odds with the hypothesis that visual areas activate less in elders than in young $[15,33,40,52,57]$. Less efficient neurophysiological processing during encoding of visual stimuli could be due to pathology in the substrates of retinotopic representation [25], spatial attention, or other aspects of encoding. Due to the spatial nature of the stimulus array, spatial attentional demands presumably increase with set size during this phase of the trial. However, spatial attention and its neural correlates do not seem to be affected by aging $[3,27,104]$.

Functionally, the $y$-intercept of fMRI signal associated with the retention delay would represent all aspects of WM that do not depend on set size. The lower neural efficiency of expression of this pattern in elders is difficult to speculate upon, as the predicted expression in the most heavily (positively) weighted voxels was negative, which thus corresponds to negative predicted $y$-intercepts in both age groups with respect to baseline levels of neural activity. Thus, more inefficient expression in the elders means that the elder activation $y$-intercepts (when normalized by performance) tended to be 
more negative than those in the young. We can say that if a reduction of set size zero neural activity from baseline is integral to retention delay processing, then elders brains manifest a more inefficient form of this mechanism than young subjects. But we nevertheless recognize the more contentious nature of comparing the intensities of deactivations, since metabolic deactivations appear to be different from inhibitory synaptic activity, which ceteris paribus should lead to an increase in metabolic activity [44].

The first latent spatial pattern associated with loaddependent retention delay processing was also less efficiently expressed in elders than young. But since the spatial activation patterns for this effect in young and elders were different, the simple neural efficiency model does not explain these data. However, it is conceivable that age-related decreases in neural efficiency of canonical brain networks and engagement of non-canonical networks are related. Consistent with this speculation, elder subjects who expressed the first latent spatial pattern the most tended to have greater expression of the second latent spatial pattern $\left(R^{2}=0.66\right)$ while the opposite was true of young subjects, with those young subjects evidencing the greatest expression of the first latent spatial pattern having expression of the second latent spatial pattern closest to zero $\left(R^{2}=0.19\right)$.

The nature of the mechanisms of reduced neural efficiency in elders could presumably be informed by cross-sectional correlations of inefficiency with age-related neuropathology, modeling of neural circuit bioenergetics, and in vivo and in vitro pharmacological studies. We feel that more needs to be done to see if the definition of neural efficiency adopted here is a useful descriptor for the effects of brain pathology in aging. For example, one obvious issue is the construct's sensitivity to the units of measurement.

\subsection{Compensatory reorganization and dedifferentiation}

Non-identity of young and elder brain activation patterns was detected for load-dependent processing associated with the retention delay. This effect is presumed to relate to load-dependent verbal WM maintenance. Thus, our findings support the existence of some age-related change in the brain networks that mediate this process that cannot be simply explained as a scaling. However, this does not mean that these networks in the young and elder populations are nonoverlapping, as the first spatial pattern was expressed with the same sign and similar magnitudes in both age groups. In contrast, the second latent spatial pattern was expressed with different signs in the two age groups, and primarily in elders. This second pattern was most heavily weighted in right parahippocampal gyrus/lingual gyrus, where elders showed positive and young subjects showed near nil load-dependent activation during the retention delay. It is important to stress that not all important elements of latent spatial pattern 2 might be above visualization thresholds, and that a more powerful study would be required to localize them with reasonable control over type II error rate.
When the second latent pattern has null expression in both age groups, then both age groups have identical spatial patterns (equal to the first latent pattern). Expression of the second latent pattern was near zero for both age groups at faster memory scanning speeds. This means that young activation patterns were more similar to those of higher performing elders than those of lower performing elders. This is not consistent with cross-sectional models of compensatory reorganization such as HAROLD [14]. In contrast, this finding is consistent with, but does not imply, that the age-related change in activation pattern is a cause of impaired performance.

This result is also consistent with the idea of neural compensation that we have discussed previously in the context of cognitive reserve [89]. According to this idea, age-related changes in activation patterns might arise when age-related brain pathology has impaired the brain networks normally used by younger individuals: The expression of these new activation patterns might be compensatory in that they could have a beneficial effect on performance relative to their theoretical absence, even if they are not associated with better task performance in a cross-sectional sense. Also, although this compensatory reorganization would arise in response to age-related brain pathology, individuals with same degree of underlying pathology might vary in their ability to compensate [89]. In order to test this interaction between brain reorganization, behavioral performance, and cognitive reserve one would need an index of brain pathology, which the current study lacks.

\subsection{Consideration of population under study}

High densities of plaques and tangles, the main pathological markers of $\mathrm{AD}$, are not a necessary consequence of aging, as the majority of elders who are cognitively intact at the time of death have minimal amounts of AD-like cortical neuropathology $[34,46,81]$. However, it is possible for a cognitively intact person to have a high degree of AD-type neuropathology [85]. As assessed by mMMSTotal [86], the population of interest in the current study comprises elders absent dementia or mild cognitive impairment [36], but we do not know positively whether these behaviorally normal elders are absent any AD pathology. The elders were also well matched with the young subjects on years of education, but tended to have somewhat lower IQ values. Future work will investigate the relationship between IQ and other hypothesized aspects of cognitive reserve on neural correlates of WM. Another caveat is that the sampling for the study was not random, with certain subpopulations of elderly (e.g., those associated with senior centers) and young (e.g., Columbia University students) being over-represented.

\subsection{Relationship to previous findings}

Greater levels of activation for elders than young subjects have been reported in some brain areas under various 
conditions [15,33,57,58,71,75]. One influential study reported greater DIR task activation (ostensibly related to $\mathrm{WM}$ ) in elders compared to younger subjects in premotor cortex, parietal cortex, and ventral and DLPFC [15]. Unfortunately there were problems in the fMRI time-series modeling of that report that makes its neuroimaging results difficult to interpret. In contrast, the strength of the current study is that it modeled several features (across trial components and set size) of the functional responses associated with DIR trials in a rational and interpretable manner.

Another study reported no effect of age on ventral PFC set size-dependent activation and greater DLPFC activation in younger than elder subjects [75]. This is quite different from our findings in that DLPFC and anterior insula (which is proximal to and could be potentially reported as ventral PFC) had substantial spatial weights in latent spatial pattern 1 of load-dependent processing during the retention delay, which was expressed to a greater degree in elders. But that study collapsed across all trial phases [75], and had other methodological differences from the current study, making them difficult to meaningfully compare.

In a different study by the same group, greater DLPFC activation was found in younger than older adults only during the probe phase of a DIR task [74], with probe phase DLPFC activation correlating negatively with RT in elders. As that study collapsed fMRI signal over set sizes, it is not easily comparable with the results from the current study. Nevertheless, there was no correlation detected between expressions of latent spatial pattern 1 of load-independent or load-dependent probe period processing with either RT slope or RT intercept in the elders (all $R^{2}$ estimates $<0.01$ ). However, there were significant correlations between RT slope and expression of latent spatial patterns $1\left(R^{2}=0.33\right)$ and $2\left(R^{2}=0.37\right)$ of loaddependent retention delay processing in the elders. But, the direction of the correlation they reported in elders collapsed over set sizes during the probe phase (slower RT being associated with smaller DLPFC activation) is opposite in sign from the one detected here (taking into account the relative signs of the spatial weights in right DLPFC and expression of latent spatial pattern 1) which had slower RT being associated with greater pattern expression. Finally, our findings of greater overall activation during the retention delay in elders go against their conclusion that age-related differences in the neural correlates of DIR task performance are limited to the probe phase.

In contrast to increases in activation in elders, there have also been many reports of reduced activations in elders $[15,32,40,41,43,57-59,74,75,79]$. We did not detect any such effects at the spatial pattern level (which would have been manifest as a lower, i.e., closer to zero, expression of a pattern in elders than young) or at the voxel-wise level using SPM. Thus this study also yielded no results consistent with the unsubtle idea that some parts of elder brains might simply stop working altogether [32].

The broader significance of the current study is that it is first time the idea has been formally tested that normal aging might simply vary the gain of neural activity in an ageindependent brain network. Vis a vis Fig. 1, greater activation detected via SPM in elders over young has tended to be interpreted as compensatory reorganization $[4,10,14,33,87,100]$, without consideration of the alternative that there is a general increase in expression of an age-independent brain activation pattern, and that what is tending to be detected are simply the largest ensuing age-dependent intensity differences. Critical to appreciating this alternative is the fact that a simple scaling (i.e., gain) difference in brain pattern expression between two groups can lead to spatially varying activation intensity differences between groups.

\subsection{Alternative explanations of current findings}

For comparisons of BOLD signal changes across groups to be neurophysiologically meaningful, the coupling of neural activity to BOLD signal must be essentially the same in the two groups. On average, the gain of coupling of neural activity to the BOLD signal has been reported to be essentially unchanged with normal aging, though there is greater within-group variability in elder subjects [24,39]. This evidence that neural-hemodynamic coupling is not changed with normal aging is critical as it allows other differences in fMRI response to be interpreted, at least tentatively, as reflective of neurophysiological differences.

The brain tends to atrophy with aging [31], and it is possible that gray matter loss is closely related to any age effects on brain activation. This is because analyses of imaging data performed in standard brain space do not typically involve normalization of the activation measures by gray matter concentration per voxel, and so a standard space voxel with low gray matter concentration would be expected ceteris paribus to have a low activation signal. Such effects have been observed [30,68,93], but not always [42]. Also, in Alzheimer's disease patients an inverse relationship between gray matter concentration in hippocampus and memory taskrelated activation in inferior frontal gyrus and cerebellum has been observed [30]. In the current experiment, total (i.e., averaged over the entire brain in standard space) elder gray matter concentration was not correlated with the expression of any of the latent spatial patterns (all two-tailed $p>0.05$ ). This does not rule out that local gray matter concentrations have relationships with activation levels. However, that elders did not tend to have lower activation levels than young subjects seems to speak against the possibility that atrophy is responsible in a direct way for any of our fMRI findings. Indeed, if fMRI signal had been normalized by GM concentrations in this study, it seems quite likely that the resulting elder activations would have been that much greater than those in young.

A reviewer raised the issue of whether differences in the quality of coregistration of structural to functional data between the two age groups could account for any of the findings. If there were increased coregistration noise in the elderly, this would be expected ceteris paribus to reduce 
observed amplitudes of fMRI activation in the elderly in the second-level GLM, which was the opposite of what tended to be observed.

\section{Conclusions}

None of the models under consideration were able to explain all of the age-related differences in brain activity during performance of a DIR task. There was evidence for neural inefficiency (load-dependent aspects of encoding and load-independent aspects of verbal WM) and dedifferentiation (or possibly compensation [89]; load-dependent aspects of verbal WM maintenance). Neither capacity limitation nor cross-sectional models of compensatory reorganization were supported for any effect examined. For the load-dependent process of memory scanning and load-independent aspects of encoding or response planning/execution, no effect of aging was detected at all. These results suggest that, even within the same task, the existence and nature of brain activation changes with aging can vary based on cognitive process engaged.

\section{Acknowledgements}

This research was supported by NIA grants RO1 AG026158 and RR00645. Eric Zarahn was supported in part by a 2002 NARSAD Young Investigator Award.

\section{References}

[1] Alvarez P, Zolamorgan S, Squire LR. The animal-model of human amnesia-long-term-memory impaired and short-termmemory intact. Proc Natl Acad Sci USA 1994;91(12):5637-41.

[2] Anders T, Fozard J, Lillyquist T. Effects of age upon retrieval from short-term memory. Dev Psychol 1972;6(2):214-7.

[3] Atchley P, Kramer AF. Spatial cuing in a stereoscopic display: attention remains "depth-aware" with age. J Gerontol B Psychol Sci Soc Sci 1998;53(5):P318-23.

[4] Backman L, Andersson JL, Nyberg L, Winblad B, Nordberg A, Almkvist $\mathrm{O}$. Brain regions associated with episodic retrieval in normal aging and Alzheimer's disease. Neurology 1999;52(9):1861-70.

[5] Baddeley A. Working memory. Science 1992;255(5044):556-9.

[6] Baddeley A, Chincotta D, Stafford L, Turk D. Is the word length effect in STM entirely attributable to output delay? Evidence from serial recognition. Q J Exp Psychol A 2002;55A(2):353-69.

[7] Baddeley AD. Working memory. New York: Oxford University Press; 1986.

[8] Barnes LL, Nelson JK, Reuter-Lorenz PA. Object-based attention and object working memory: overlapping processes revealed by selective interference effects in humans. Prog Brain Res 2001;134:471-81.

[9] Baudouin A, Vanneste S, Isingrini M. Age-related cognitive slowing: the role of spontaneous tempo and processing speed. Exp Aging Res 2004;30(3):225-39.

[10] Becker JT, Mintun MA, Aleva K, Wiseman MB, Nichols T, DeKosky ST. Compensatory reallocation of brain resources supporting verbal episodic memory in Alzheimer's disease. Neurology 1996;46(3):692-700.
[11] Belleville S, Peretz I, Arguin M. Contribution of articulatory rehearsal to short-term memory: evidence from a case of selective disruption. Brain Lang 1992;43(4):713-46.

[12] Brody H. The nervous system and aging. Adv Pathobiol 1980;7: 200-9.

[13] Cabeza R. Hemispheric asymmetry reduction in older adults: the HAROLD model. Psychol Aging 2002;17(1):85-100.

[14] Cabeza R, Anderson ND, Locantore JK, McIntosh AR. Aging gracefully: compensatory brain activity in high-performing older adults. Neuroimage 2002;17(3):1394-402.

[15] Cabeza R, Daselaar SM, Dolcos F, Prince SE, Budde M, Nyberg L. Task-independent and task-specific age effects on brain activity during working memory, visual attention and episodic retrieval. Cereb Cortex 2004;14(4):364-75.

[16] Callicott J, Bertolino A, Mattay V, Langheim F, Duyn J, Coppola R, et al. Physiological dysfunction of the dorsolateral prefrontal cortex in schizophrenia revisited. Cereb Cortex 2000;10(11):1078-92.

[17] Chaytor N, Schmitter-Edgecombe M. Working memory and aging: a cross-sectional and longitudinal analysis using a self-ordered pointing task. J Int Neuropsychol Soc 2004;10(4):489-503.

[18] Christensen H, Mackinnon AJ, Korten AE, Jorm AF, Henderson AS, Jacomb P, et al. An analysis of diversity in the cognitive performance of elderly community dwellers: individual differences in change scores as a function of age. Psychol Aging 1999;14(3): 365-79.

[19] Cohen J, MacWhinney B, Flatt M, Provost J. PsyScope: an interactive graphic system for designing and controlling experiments in the psychology laboratory using Macintosh computers. Behav Res Methods, Instrum Comput 1993;25(2):257-71.

[20] Cohen MS, DuBois RM. Stability, repeatability, and the expression of signal magnitude in functional magnetic resonance imaging. J Magn Reson Imaging 1999;10(1):33-40.

[21] Coleman PD, Flood DG. Neuron numbers and dendritic extent in normal aging and Alzheimer's disease. Neurobiol Aging 1987;8(6): 521-45.

[22] Cowan N, Nugent LD, Elliott EM, Geer T. Memory-search and rehearsal processes and the word length effect in immediate recall: a synthesis in reply to Service. Q J Exp Psychol A 2000;53A(3): 666-70.

[23] De Beni R, Palladino P. Decline in working memory updating through ageing: intrusion error analyses. Memory 2004;12(1): $75-89$.

[24] D'Esposito M, Zarahn E, Aguirre G, Rypma B. The effect of normal aging on the coupling of neural activity to the BOLD hemodynamic response. Neuroimage 1999;10(1):6-14.

[25] Devaney KO, Johnson HA. Neuron loss in the aging visual cortex of man. J Gerontol 1980;35(6):836-41.

[26] Eriksen C, Hamlin R, Daye C. Aging adults and rate of memory scan. Psychon Bull Rev 1973;1(4):259-60.

[27] Folk CL, Hoyer WJ. Aging and shifts of visual spatial attention. Psychol Aging 1992;7(3):453-65.

[28] Friston KJ, Holmes A, Poline JB, Price CJ, Frith CD. Detecting activations in PET and fMRI: levels of inference and power. Neuroimage 1996;4(3 Pt 1):223-35.

[29] Friston KJ, Stephan KE, Lund TE, Morcom A, Kiebel S. Mixedeffects and fMRI studies. Neuroimage 2005;24(1):244-52.

[30] Garrido GE, Furuie SS, Buchpiguel CA, Bottino CM, Almeida OP, Cid CG, et al. Relation between medial temporal atrophy and functional brain activity during memory processing in Alzheimer's disease: a combined MRI and SPECT study [see comment]. J Neurol Neurosurg Psychiatry 2002;73(5):508-16.

[31] Good CD, Johnsrude IS, Ashburner J, Henson RNA, Friston KJ, Frackowiak RSJ. A voxel-based morphometric study of ageing in 465 normal adult human brains. Neuroimage 2001;14(1): 21-36.

[32] Grady CL, McIntosh AR, Horwitz B, Maisog JM, Ungerleider LG, Mentis MJ, et al. Age-related reductions in human recogni- 
tion memory due to impaired encoding. Science 1995;269(5221): 218-21.

[33] Grady CL. Age-related changes in cortical blood flow activation during perception and memory. Ann NY Acad Sci 1996;777:14-21.

[34] Green MS, Kaye JA, Ball MJ. The Oregon brain aging study: neuropathology accompanying healthy aging in the oldest old. Neurology 2000;54(1):105-13.

[35] Greene HA, Madden DJ. Adult age differences in visual acuity, stereopsis, and contrast sensitivity. Am J Optom Physiol Opt 1987;64(10):749-53.

[36] Grundman M, Petersen RC, Ferris SH, Thomas RG, Aisen PS, Bennett DA, et al. Mild cognitive impairment can be distinguished from Alzheimer disease and normal aging for clinical trials. Arch Neurol 2004;61(1):59-66.

[37] Hecker R, Mapperson B. Dissociation of visual and spatial processing in working memory. Neuropsychologia 1997;35(5):599-603.

[38] Holmes A, Friston K. Generalisability, random effects and population inference. Neuroimage 1998;7:S754.

[39] Huettel SA, Singerman JD, McCarthy G. The effects of aging upon the hemodynamic response measured by functional MRI. Neuroimage 2001;13(1):161-75.

[40] Iidaka T, Sadato N, Yamada H, Murata T, Omori M, Yonekura Y. An fMRI study of the functional neuroanatomy of picture encoding in younger and older adults. Brain Res Cogn Brain Res 2001;11(1):111.

[41] Johnson MK, Mitchell KJ, Raye CL, Greene EJ. An age-related deficit in prefrontal cortical function associated with refreshing information. Psychol Sci 2004;15(2):127-32.

[42] Johnson SC, Saykin AJ, Baxter LC, Flashman LA, Santulli RB, McAllister TW, et al. The relationship between fMRI activation and cerebral atrophy: comparison of normal aging and Alzheimer disease. Neuroimage 2000;11(3):179-87.

[43] Jonides J, Marshuetz C, Smith EE, Reuter-Lorenz PA, Koeppe RA, Hartley A. Age differences in behavior and PET activation reveal differences in interference resolution in verbal working memory. $\mathbf{J}$ Cogn Neurosci 2000;12(1):188-96.

[44] Jueptner M, Weiller C. Review: does measurement of regional cerebral blood flow reflect synaptic activity?-implications for PET and fMRI. Neuroimage 1995;2:148-56.

[45] Kirschen MP, Chen SH, Schraedley-Desmond P, Desmond JE. Load- and practice-dependent increases in cerebro-cerebellar activation in verbal working memory: an fMRI study. Neuroimage 2005;24(2):462-72.

[46] Knopman DS, Parisi JE, Salviati A, Floriach-Robert M, Boeve BF, Ivnik RJ, et al. Neuropathology of cognitively normal elderly. J Neuropathol Exp Neurol 2003;62(11):1087-95.

[47] Kray J, Lindenberger U. Adult age differences in task switching. Psychol Aging 2000;15(1):126-47.

[48] Kwong KK, Belliveau JW, Chesler DA, Goldberg IE, Weisskoff RM, Poncelet BP, et al. Dynamic magnetic resonance imaging of human brain activity during primary sensory stimulation. Proc Natl Acad Sci 1992;89(12):5675-9.

[49] Light LL, Anderson PA. Working-memory capacity, age, and memory for discourse. J Gerontol 1985;40(6):737-47.

[50] Lindenberger U, Scherer H, Baltes PB. The strong connection between sensory and cognitive performance in old age: not due to sensory acuity reductions operating during cognitive assessment. Psychol Aging 2001;16(2):196-205.

[51] Longoni AM, Richardson JT, Aiello A. Articulatory rehearsal and phonological storage in working memory. Mem Cognit 1993;21(1):11-22.

[52] Madden DJ, Turkington TG, Coleman RE, Provenzale JM, DeGrado TR, Hoffman JM. Adult age differences in regional cerebral blood flow during visual world identification: evidence from H215O PET. Neuroimage 1996;3(2):127-42.

[53] Madden DJ, Turkington TG, Provenzale JM, Denny LL, Hawk TC, Gottlob LR, et al. Adult age differences in the functional neuroanatomy of verbal recognition memory. Hum Brain Mapp 1999;7(2):115-35.

[54] Manoach D, Gollub R, Benson E, Searl M, Goff D, Halpern E, et al. Schizophrenic subjects show aberrant fMRI activation of dorsolateral prefrontal cortex and basal ganglia during working memory performance. Biol Psychiatry 2000;48(2):99-109.

[55] Manoach DS, Schlaug G, Siewert B, Darby DG, Bly BM, Benfield A, et al. Prefrontal cortex fMRI signal changes are correlated with working memory load. Neuroreport 1997;8(2):545-9.

[56] McIntosh AR, Bookstein FL, Haxby JV, Grady CL. Spatial pattern analysis of functional brain images using partial least squares. Neuroimage 1996;3(3 Pt 1):143-57.

[57] Mencl WE, Pugh KR, Shaywitz SE, Shaywitz BA, Fulbright RK, Constable RT, et al. Network analysis of brain activations in working memory: behavior and age relationships. Microsc Res Technol 2000;51(1):64-74.

[58] Milham MP, Erickson KI, Banich MT, Kramer AF, Webb A, Wszalek $\mathrm{T}$, et al. Attentional control in the aging brain: insights from an fMRI study of the stroop task. Brain Cogn 2002;49(3):277-96.

[59] Mitchell KJ, Johnson MK, Raye CL, D'Esposito M. fMRI evidence of age-related hippocampal dysfunction in feature binding in working memory. Brain Res Cogn Brain Res 2000;10(1/2):197-206.

[60] Morcom AM, Good CD, Frackowiak RS, Rugg MD. Age effects on the neural correlates of successful memory encoding. Brain 2003;126(Pt 1):213-29.

[61] Neubauer AC, Grabner RH, Freudenthaler HH, Beckmann JF, Guthke $\mathrm{J}$. Intelligence and individual differences in becoming neurally efficient. Acta Psychologica 2004;116(1):55-74.

[62] Nielsen-Bohlman L, Knight RT. Prefrontal alterations during memory processing in aging. Cereb Cortex 1995;5(6):541-9.

[63] Ogawa S, Menon RS, Tank DW, Kim SG, Merkle H, Ellermann JM, et al. Functional brain mapping by blood oxygenation level-dependent contrast magnetic resonance imaging. A comparison of signal characteristics with a biophysical model. Biophys J 1993;64(3):80312.

[64] Orsini A, Grossi D, Capitani E, Laiacona M, Papagno C, Vallar G. Verbal and spatial immediate memory span: normative data from 1355 adults and 1112 children. Ital J Neurol Sci 1987;8(6):53948.

[65] Perl DP, Pendlebury WW. Neuropathology of dementia. Neurol Clin 1986;4(2):355-68.

[66] Ramsey NF, Koning HA, Welles P, Cahn W, van der Linden JA, Kahn RS. Excessive recruitment of neural systems subserving logical reasoning in schizophrenia. Brain 2002;125(Pt 8):1793-807.

[67] Ranganath C, Yonelinas AP, Cohen MX, Dy CJ, Tom SM, D'Esposito M. Dissociable correlates of recollection and familiarity within the medial temporal lobes. Neuropsychologia 2004;42(1):2-13.

[68] Remy F, Mirrashed F, Campbell B, Richter W. Verbal episodic memory impairment in Alzheimer's disease: a combined structural and functional MRI study. Neuroimage 2005;25(1):253-66.

[69] Reuter-Lorenz PA, Jonides J, Smith EE, Hartley A, Miller A, Marshuetz C, et al. Age differences in the frontal lateralization of verbal and spatial working memory revealed by PET. J Cogn Neurosci 2000;12(1):174-87.

[70] Roberts TP, Disbrow EA, Roberts HC, Rowley HA. Quantification and reproducibility of tracking cortical extent of activation by use of functional MR imaging and magnetoencephalography [see comment]. AJNR Am J Neuroradiol 2000;21(8):1377-87.

[71] Rosen AC, Prull MW, O'Hara R, Race EA, Desmond JE, Glover GH, et al. Variable effects of aging on frontal lobe contributions to memory. Neuroreport 2002;13(18):2425-8.

[72] Rypma B, D'Esposito M. The roles of prefrontal brain regions in components of working memory: effects of memory load and individual differences. Proc Natl Acad Sci USA 1999;96(11):6558-63.

[73] Rypma B, Prabhakaran V, Desmond JE, Glover GH, Gabrieli JDE. Load-dependent roles of frontal brain regions in the maintenance of working memory. Neuroimage 1999;9(2):216-26. 
[74] Rypma B, D'Esposito M. Isolating the neural mechanisms of age-related changes in human working memory. Nat Neurosci 2000;3(5):509-15.

[75] Rypma B, Prabhakaran V, Desmond JE, Gabrieli JD. Age differences in prefrontal cortical activity in working memory. Psychol Aging 2001;16(3):371-84.

[76] Rypma B, Berger JS, D'Esposito M. The influence of workingmemory demand and subject performance on prefrontal cortical activity. J Cogn Neurosci 2002;14(5):721-31.

[77] Saad ZS, Ropella KM, DeYoe EA, Bandettini PA. The spatial extent of the BOLD response. Neuroimage 2003;19(1):132-44.

[78] Salthouse TA. Memory aging from 18 to 80. Alzheimer Dis Assoc Disord 2003;17(3):162-7.

[79] Schacter DL, Savage CR, Alpert NM, Rauch SL, Albert MS. The role of hippocampus and frontal cortex in age-related memory changes: a PET study. Neuroreport 1996;7(6):1165-9.

[80] Schmidt R, Schmidt H, Kapeller P, Lechner A, Fazekas F. Evolution of white matter lesions. Cerebrovasc Dis 2002;13(Suppl 2):16-20.

[81] Schmitt FA, Davis DG, Wekstein DR, Smith CD, Ashford JW, Markesbery WR. "Preclinical" AD revisited: neuropathology of cognitively normal older adults. Neurology 2000;55(3):370-6.

[82] Shah P, Miyake A. The separability of working memory resources for spatial thinking and language processing: an individual differences approach. J Exp Psychol Gen 1996;125(1):4-27.

[83] Silver MH, Newell K, Brady C, Hedley-White ET, Perls TT. Distinguishing between neurodegenerative disease and disease-free aging: correlating neuropsychological evaluations and neuropathological studies in centenarians. Psychosom Med 2002;64(3):493-501.

[84] Simic G, Kostovic I, Winblad B, Bogdanovic N. Volume and number of neurons of the human hippocampal formation in normal aging and Alzheimer's disease. J Comp Neurol 1997;379(4): 482-94.

[85] Snowdon DA, Nun S. Healthy aging and dementia: findings from the Nun Study. Ann Intern Med 2003;139(5 Pt 2):450-4.

[86] Stern Y, Sano M, Paulson J, Mayeux R. Modified mini-mental state examination: validity and reliability. Neurology 1982;37(Suppl 1): 179 .

[87] Stern Y, Moeller JR, Anderson KE, Luber B, Zubin NR, DiMauro AA, et al. Different brain networks mediate task performance in normal aging and AD: defining compensation. Neurology 2000;55(9):1291-7.

[88] Stern Y, Zarahn E, Hilton HJ, Flynn J, DeLaPaz R, Rakitin B. Exploring the neural basis of cognitive reserve. J Clin Exp Neuropsychol 2003;25(5):691-701

[89] Stern Y, Habeck C, Moeller J, Scarmeas N, Anderson KE, Hilton $\mathrm{HJ}$, et al. Brain networks associated with cognitive reserve in healthy young and old adults. Cereb Cortex 2005;15(4):394-402.

[90] Sternberg S. High-speed scanning in human memory. Science 1966;153(736):652-4.

[91] Sturgis R, Lemke EA, Johnson JJ. A validity study of the neural efficiency analyzer in relation to selected measures of intelligence. Percept Mot Skills 1977;45(2):475-8.
[92] Talairach J, Tournoux P. Co-planar stereotactic atlas of the human brain. Three-dimensional proportional system: an approach of cerebral imaging. New York: Theime Medical Publisher; 1988.

[93] Thomsen T, Specht K, Rimol LM, Hammar A, Nyttingnes J, Ersland $\mathrm{L}$, et al. Brain localization of attentional control in different age groups by combining functional and structural MRI. [Erratum appears in Neuroimage 2005;25(1) 332. Note: Rimol Lars Morten [added]]. Neuroimage 2004;22(2):912-9.

[94] Tresch MC, Sinnamon HM, Seamon JG. Double dissociation of spatial and object visual memory: evidence from selective interference in intact human subjects. Neuropsychologia 1993;31(3): 211-9.

[95] Vallar G, Baddeley AD. Fractionation of working memoryneuropsychological evidence for a phonological short-term store. J Verbal Learn Verbal Behav 1984;23(2):151-61.

[96] Veltman DJ, Rombouts S, Dolan RJ. Maintenance versus manipulation in verbal working memory revisited: an fMRI study. Neuroimage 2003;18(2):247-56.

[97] Verhaeghen P, Salthouse TA. Meta-analyses of age-cognition relations in adulthood: estimates of linear and nonlinear age effects and structural models. Psychol Bull 1997;122(3):231-49.

[98] Warrington EK, Shallice T. The selective impairment of auditory verbal short-term memory. Brain 1969;92(4):885-96.

[99] West MJ. Regionally specific loss of neurons in the aging human hippocampus. Neurobiol Aging 1993;14(4):287-93.

[100] Woodard JL, Grafton ST, Votaw JR, Green RC, Dobraski ME, Hoffman JM. Compensatory recruitment of neural resources during overt rehearsal of word lists in Alzheimer's disease. Neuropsychology 1998;12(4):491-504.

[101] Worsley KJ. Local maxima and the expected Euler characteristic of excursion sets of chi-squared, $F$ and $t$ fields. Adv Appl Prob 1994;26(1):13-42.

[102] Worsley KJ, Poline JB, Vandal AC, Friston KJ. Tests for distributed, nonfocal brain activations. Neuroimage 1995;2(3):183-94.

[103] Worsley KJ, Poline JB, Friston KJ, Evans AC. Characterizing the response of PET and fMRI data using multivariate linear models. Neuroimage 1997;6(4):305-19.

[104] Yamaguchi S, Tsuchiya H, Kobayashi S. Electrophysiologic correlates of age effects on visuospatial attention shift. Brain Res Cogn Brain Res 1995;3(1):41-9.

[105] Zarahn E. Testing for neural responses during temporal components of trials with BOLD fMRI. Neuroimage 2000;11(6 Pt 1):78396.

[106] Zarahn E, Rakitin B, Abela D, Flynn J, Stern Y. Positive evidence against human hippocampal involvement in working memory maintenance of familiar stimuli. Cereb Cortex 2005;15(3):30316.

[107] Zola-Morgan S, Squire L, Amaral D, Suzuki W. Lesions of perirhinal and parahippocampal cortex that spare the amygdala and hippocampal formation produce severe memory impairment. J Neurosci 1989;9(43):55-70. 